\title{
Night-time work and all-cause mortality in the general working population of Denmark
}

\author{
Harald Hannerz ${ }^{1}$ Helle Soll-Johanning ${ }^{1} \cdot$ Ann Dyreborg Larsen $^{1}\left[\right.$ (D) Anne Helene Garde ${ }^{1,2}$
}

Received: 30 May 2018 / Accepted: 1 December 2018 / Published online: 4 December 2018

(c) The Author(s) 2018

\begin{abstract}
Purpose A recent study among female nurses in Denmark found an increased mortality among night-time workers, which has raised concerns about the sufficiency of the EU Working Time Directive. The aim of the present study was to examine the relationship between night-time work and all-cause mortality among full-time employees in the general workforce of Denmark.

Methods Interview data from the Danish Labour Force Surveys, 1999-2013, were linked to national registers with individual-level data on occupation, industry, socioeconomic status (SES), emigrations and deaths. The participants $(N=159,933)$ were followed from the end of the calendar year of the interview until the end of 2014. Poisson regression was used to estimate rate ratios for all-cause mortality, with and without stratification by sex and socioeconomic status. A likelihood ratio test was used to test the overall null-hypothesis, which stated that the mortality rates were independent of night-time work, SES $\times$ night-time work and sex $\times$ night-time work.

Results The likelihood ratio test did not reject the null hypothesis $(p=0.14)$. The rate ratio for all-cause mortality among employees with vs. without night-time work was estimated at 1.07 (95\% CI 0.97-1.19) after adjustment for age, sex, SES, calendar time, weekly working hours and time passed since the start of follow-up.

Conclusions The present study did not find any statistically significant associations between night-time work and all-cause mortality among employees in the general workforce of Denmark.
\end{abstract}

Keywords EU Working Time Directive $\cdot$ Hypothesis testing · Occupational health · Cohort study

$\begin{array}{ll}\text { Abbreviations } \\ \text { CI } & \text { Confidence interval } \\ \text { DLFS } & \text { The Danish Labour Force Survey } \\ \text { ESeC } & \text { The European Socio-economic Classification } \\ \text { EU } & \text { European Union } \\ \text { RR } & \text { Rate ratio } \\ \text { SES } & \text { Socio-economic status }\end{array}$

Ann Dyreborg Larsen adl@nrcwe.dk

1 National Research Centre for the Working Environment, Copenhagen, Denmark

2 Department of Public Health, University of Copenhagen, Copenhagen, Denmark

\section{Background}

Night-time work has been associated with insufficient sleep as well as a wide range of safety and health problems (Itani and Kaneita 2016; Kecklund and Axelsson 2016). To protect the safety and health of night-shift workers, the EU Working Time Directive (Smet 1993) therefore, stipulates that the Member States shall take the measures necessary to ensure that night workers work no more than $8 \mathrm{~h}$ during a $24-\mathrm{h}$ period, are offered a free health assessment and have access to appropriate health and safety protection.

If the intent of the directive is fulfilled, then the nighttime regulations should be sufficient to protect against adverse health effects from night-time work. However, a recent study found an association between night-time work and all-cause mortality among female nurses in Denmark (Jorgensen et al. 2017). They reported an age-standardised rate ratio of 1.74 (95\% CI 1.48-2.07) among nurses who normally work at night, compared with nurses who normally work during the day (Jorgensen et al. 2017). A similar 
phenomenon has been observed with all-cause mortality rates among nurses in USA ( $\mathrm{Gu}$ et al. 2015). In their study, the rates were higher for women with 6-14 years experience with night-shift work (HR 1.11, 95\% CI 1.06-1.17) and more than 15 years (HR 1.11, 95\% CI 1.05-1.18), compared to women who never worked night shifts ( $\mathrm{Gu}$ et al. 2015). The question is if this should be regarded as an isolated problem, which only concerns (female) health care workers, or as a general problem which concerns all night workers in the Danish labour force. When not focusing on female nurses, studies are inconclusive. In a meta-analysis by Lin et al. (2015), the authors observed an increased risk of cause-specific mortality (cancer, cardiovascular events), but for all-cause mortality the estimates were statistically insignificant (RR 1.25, 95\% CI 0.79-2.00).

\section{Aim}

The aim of the present study was to investigate if night-time work is associated with all-cause mortality rates among fulltime employees in Denmark, either as a main (general) effect or as an effect of interaction with socioeconomic status or sex.

\section{Methods}

The material of the study is described in a protocol by Hannerz et al. (2016). The methods of the study are detailed in another study protocol, which was published before we looked at any relation between the exposure and the outcome data of the study (Hannerz and Soll-Johanning 2017). Relevant details of these protocols will be repeated in the present method section.

\section{Study population}

"The Danish Labour Force Survey (DLFS) is based on quarterly random samples of 15-74-year-old people in the Danish population" (Hannerz and Soll-Johanning 2017). The samples are drawn from the Central Person Register which, inter alia, covers information on addresses, sex, and dates of birth, death, and migrations for every person who is or has been an inhabitant of Denmark sometime between 1968 and the present (Pedersen 2011). "Each participant is invited to be interviewed 4 times over a period of one and a half years" (Hannerz and Soll-Johanning 2017). Person-based information on, inter alia, employment status and working time arrangements is collected through structured telephone interviews (Statistics Denmark 2015). Calls are made from 9 a.m. till 9 p.m. on weekdays, from 11 a.m. till 5 p.m. on
Saturdays and from 11 a.m. till 6 p.m. on Sundays. The response rate has decreased with time, from $70 \%$ in 2002 to $53 \%$ in 2013.

Individuals who participated in DLFS sometime during the time period 1999-2013 were eligible for inclusion in the present study if they fulfilled the following criteria:

- They were alive, 20-64 years old and residents in Denmark at the end of the calendar year of their first interview.

- They were employed with a usual work week of 32-100 h at the time of their first interview.

- They had a valid response to the question about nighttime work

In total, 357,085 people participated in at least one interview in the concerned time span. 69,221 persons were excluded due to the age criteria, 87,960 were excluded due to the employment status criteria, 38,413 were excluded for working less than $32 \mathrm{~h}$ a week, 322 were excluded for reporting to work more than $100 \mathrm{~h}$ a week, 473 were excluded due to death or emigration before the end of the calendar year of the interview, 11 were excluded for not being found in national registers, and 752 were excluded due to missing value on night-time work, which left 159,933 persons to be included in the analysis of the present study.

\section{Exposure to night-time work}

The questions used to obtain information about night-time work have changed slightly with time. Before 2001, the participants were simply asked "Do you work at night?" with the following response categories: "yes, regularly", "yes, occasionally", and "no, never". From 2001 and onward the question was changed to "Did you work at night sometime during the last four weeks?", and from 2007 onward the response categories were expanded to "yes, regularly" (i.e. more than half of the working days in the last 4 weeks), "yes occasionally" (i.e. at least once within the last 4 weeks, but less than half of the working days), and "no, not within the last 4 weeks".

In the present study, the participants who responded with either "yes, regularly" or "yes, occasionally" were defined as exposed while those who responded with "no..." were defined as unexposed to night-time work.

\section{Follow-up}

The included participants were followed from the beginning of the calendar year that succeeds the year of their baseline interview. The follow-up ended at the time they emigrated or died, or the study period ended (December 31, 2014), whichever came first. Dates of death and emigration were 
ascertained through the Central Person Register (Pedersen 2011).

\section{Covariates}

The following covariates were included in the analyses: Night-time work, sex, age (10-year classes), usual weekly working hours (32-40, 41-48, or $>48 \mathrm{~h}$ a week), calendar time (2000-2004, 2005-2009, or 2010-2014), time passed since start of follow-up $(0-4,5-9$, or $\geq 10$ years), and SES (low, medium, high, or unknown). Age, calendar time, and time passed since the start of follow-up were treated as dynamic (time-varying) variables. The remaining variables were fixed at the calendar year of the interview.

The participant's main occupation and industry during the calendar year of the interview were retrieved from the Employment Classification Module, which covers all inhabitants of Denmark (Petersson et al. 2011). SES was thereafter determined on the basis of the participant's occupation in accordance with the three-class version of the European Socio-economic Classification (ESeC). The coding procedure is given by Hannerz et al. (Hannerz et al. 2016).

\section{Primary statistical analysis}

We used Poisson regression to analyse all-cause mortality rates as a function of the above covariates. The logarithm of person-years at risk was used as offset and the maximum likelihood method was used to estimate the parameters. Our main interest was to see if we could reproduce the results from the study of female nurses; which is why the main analyses include the interaction terms SES $\times$ night-time work and sex $\times$ night-time work.

The following null-hypotheses were tested:

1. All-cause mortality rates among full-time employees in Denmark are independent of night-time, SES $\times$ nighttime work and sex $\times$ night-time work.

1.1 The association between night-time work and allcause mortality is independent SES.

1.2 The association between night-time work and allcause mortality is independent of sex.

1.3 The all-cause mortality rates are independent of night-time work when we disregard interaction effects.

To ascertain that the probability of a chance finding would be less than 5\%, we used the following testing hierarchy:

First level-main analyses including the interaction terms SES $\times$ night-time work and sex $\times$ night-time work A null-hypothesis at the first level would be rejected if the $p$ value of its statistical test was less than or equal to $0.05 \mathrm{~s}$ level-without one or both interaction terms:

A null-hypothesis at the second level would be rejected if (i) its associated first-level null-hypothesis (results for main analysis) was rejected and (ii) the $p$ value of its statistical test was less than or equal to 0.05 .

The first model contained the following covariates: age, sex, calendar time, time passed since the start of follow-up and weekly working hours. The second model contained the covariates of model 1 and night-time work. The third model contained the covariates of model 2 and SES $\times$ night-time work. The fourth model contained the covariates of model 2 and sex $\times$ night-time work. The fifth model contained the covariates of model 4 and SES $\times$ night-time work.

$p$ values were obtained through likelihood ratio tests. To check the statistical significance level, the overall hypothesis test compared model 5 to model 1, i.e. the full model vs. the model without any night work parameters. The test for interaction between SES and night-time work compared model 5 to model 4 . The test for interaction between sex and night-time work compared model 5 to model 3 . The test for a main effect compared model 2 to model 1 .

Parameter estimates were used to obtain rate ratios and 95\% CIs for all-cause mortality as a function of night-time work, with and without stratification by sex and SES. Rate ratios by sex were based on the parameter estimates of model 4 , rate ratios by SES were based on the parameter estimates of model 3 and the rate ratio without stratification by sex and SES was based on the parameter estimates of model 2.

\section{Sensitivity analyses according to the study protocol}

Sensitivity analysis 1-only stable exposure To find out if the strength of the association between night-time work and all-cause mortality increases when exposure is more stable over time, we conducted a sensitivity analysis, in which we only included people who (1) participated in more than one interview, (2) were between 20 and 64 years old during their last interview, (3) were employed according to their first as well as their last interview, and (4) belonged to the same category in relation to night-time work (yes vs. no) during their last interview as they did during their first interview. The follow-up of this sensitivity analysis started on January 1 of the calendar year which succeeds the calendar year of the participant's last interview.

Sensitivity analysis 2-occasional vs. regular nighttime work In the primary analysis, we used a dichotomised variable ("yes" versus "no") to describe the participants' night-time work status. In this sensitivity analysis, we divided night-time work into three categories ("no"; "yes, 
occasionally"; "yes, regularly") and estimated the rate ratios for "yes, occasionally" and "yes, regularly" vs "no".

Sensitivity analysis 3-stratification by interview calendar period Since the questions used to obtain information about night-time work were revised in 2001 and then again in 2007, we performed a sensitivity analysis where the results were stratified by calendar period of interview (1999-2000, 2001-2006, and 2007-2013). Here it should be noted that calendar period of interview is not the same as our dynamic (time-varying) variable "calendar time", which is included as a covariate in all of our analyses.

The above sensitivity analyses did not include any interaction effects. The statistical model, covariates and inclusion criteria were otherwise the same as in model 2 of the primary analysis.

\section{Post-hoc sensitivity analyses}

1. $47 \%$ of the female night workers, $39 \%$ of the night workers among the participants in the highest social status group and $30 \%$ of the night workers in the intermediate social status group were employed in the health care sector, nursing homes or similar institutions. Since previous research indicated an effect of night-time work in the Danish health care sector (Jorgensen et al. 2017), we wanted to know if the estimated relations between night-time work and all-cause mortality in the general working population would change if the health care and nursing home workers were excluded from the analysis. $\mathrm{We}$, therefore, re-estimated the rate ratios of the primary analysis in a post-hoc sensitivity analysis which excluded these workers.

2. We wanted to know if the effect of night-time work among employees in health care, nursing homes and similar institutions differed from that among employees in other sectors. To shed some light on this issue, we estimated the rate ratio for all-cause mortality as a function of night-time work stratified by "health care, nursing homes and similar institutions" vs. "other sectors"

3. The primary analysis did not include employees who worked less than $32 \mathrm{~h}$ a week. There are, however, many night workers in the health care sector whose standard work schedules (e.g. 7 night shifts, 7 days off-duty) imply an average of only 28 working hours a week (Hedegaard 2014). We wanted to know if the estimated effect of night-time work would change if our full-time inclusion criterion changed from 32 to $100 \mathrm{~h}$ a week to 28-100 $\mathrm{h}$ a week. We, therefore, repeated the procedure of post-hoc analysis 2 with a redefined inclusion criterion at $28-100 \mathrm{~h}$ a week.

Employment in the health care sector, nursing homes and similar institutions were identified through the industrial codes
851 and 8531 according to the Danish industrial classifications DB93 (Statistics Denmark 1996) and DB03 (Statistics Denmark 2002), and the codes 86-87 according to DB07 (Statistics Denmark 2007).

\section{Results}

The primary analysis included 159,933 participants, whereof $46.2 \%$ were women and $12.6 \%$ (9.2\% of the women and $15.2 \%$ of the men) were categorised as exposed to nighttime work. The occurrence of night-time work was also dependent on SES, with 12.9, 5.4, 14.6 and $16.4 \%$ among employees with high, intermediate, low and unknown status, respectively. A total of 3374 deaths were observed during an average follow-up of 7.7 years, with a crude mortality rate (deaths per 1000 person-years at risk) at 3.5 and 1.8 among the men and women, respectively. Descriptive statistics are given in Table 1.

The main hypothesis test, which compared the full model (model 5) with the model without any night work parameters (model 1), yielded a $p$ value at 0.14 . Hence we could not reject the null-hypothesis, which stated that all-cause mortality rates among full-time employees in Denmark are independent of night-time work, SES $\times$ night-time work and

Table 1 Number of participants stratified by night-time work (yes/no) and age, sex, socioeconomic status (SES) and weekly working hours, respectively

\begin{tabular}{|c|c|c|c|c|c|}
\hline & \multirow{3}{*}{$\begin{array}{l}\text { Total } \\
N\end{array}$} & \multicolumn{4}{|c|}{ Night-time work } \\
\hline & & \multicolumn{2}{|l|}{ Yes } & \multicolumn{2}{|l|}{ No } \\
\hline & & $N$ & $\%$ & $N$ & $\%$ \\
\hline Total & 145,861 & 122,718 & 84.1 & 14,498 & 9.9 \\
\hline \multicolumn{6}{|l|}{ Age (years) } \\
\hline $20-29$ & 24,924 & 3132 & 12.6 & 21,792 & 87.4 \\
\hline $30-39$ & 40,342 & 5605 & 13.9 & 34,737 & 86.1 \\
\hline $40-49$ & 43,630 & 5838 & 13.4 & 37,792 & 86.6 \\
\hline $50-59$ & 41,542 & 4637 & 11.2 & 36,905 & 88.8 \\
\hline $60-64$ & 9495 & 865 & 9.1 & 8630 & 90.9 \\
\hline \multicolumn{6}{|l|}{ Sex } \\
\hline Men & 86,012 & 13,304 & 15.5 & 72,708 & 84.5 \\
\hline Women & 73,921 & 6773 & 9.2 & 67,148 & 90.8 \\
\hline \multicolumn{6}{|l|}{ SES } \\
\hline High & 47,300 & 6118 & 12.9 & 41,182 & 87.1 \\
\hline Medium & 29,647 & 1599 & 5.4 & 28,048 & 94.6 \\
\hline Low & 65,826 & 9600 & 14.6 & 56,226 & 85.4 \\
\hline Unknown & 17,160 & 2760 & 16.1 & 14,400 & 83.9 \\
\hline \multicolumn{6}{|c|}{ Weekly working hours } \\
\hline $32-40$ & 134,793 & 15,060 & 11.2 & 119,733 & 88.8 \\
\hline $41-48$ & 15,643 & 2216 & 14.2 & 13,427 & 85.8 \\
\hline$>48$ & 9497 & 2801 & 29.5 & 6696 & 70.5 \\
\hline
\end{tabular}


sex $\times$ night-time work. Since the first-level null-hypothesis could not be rejected, the second level hypotheses tests were redundant, according to our statistical significance criteria, i.e. none of the results of the present study is statistically significant. The results will, however, be presented for exploratory purposes, and also for the purpose of avoiding publication bias. If, for example, sex-stratified analyses were to be published when the interaction effect between the concerned exposure and sex is statistically significant but not when the interaction effect is non-significant then the sex-stratified rate ratios in the research literature would be biased away from unity.

The test for interaction yielded a $p$ value at 0.09 for SES $\times$ night-time work (model 5 vs. model 4 ) and 0.81 for sex $\times$ night-time work (model 5 vs. model 3 ). The test for a main effect on all-cause mortality among employees with vs. without night-time work (model 2 vs. model 1) yielded a $p$ value at 0.16 . The estimated rate ratios of the primary analyses are given in Table 2, with and without stratification by sex and SES, respectively.

Table 2 Rate ratio for mortality among employees in Denmark 2000-2014 (primary analyses)

\begin{tabular}{|c|c|c|c|c|c|c|}
\hline Population & $\begin{array}{l}\text { Night-time } \\
\text { work }\end{array}$ & Persons & Person years & Cases & Rate ratio & $95 \% \mathrm{CI}$ \\
\hline \multirow[t]{2}{*}{ All workers ${ }^{\mathrm{a}}$} & Yes & 20,077 & 159,584 & 464 & 1.07 & $0.97-1.19$ \\
\hline & No & 139,856 & $1,078,417$ & 2910 & 1.00 & - \\
\hline \multirow[t]{2}{*}{ Male workers ${ }^{\mathrm{b}}$} & Yes & 13,304 & 105,692 & 366 & 1.07 & $0.95-1.20$ \\
\hline & No & 72,708 & 557,249 & 1972 & 1.00 & - \\
\hline \multirow[t]{2}{*}{ Female workers $^{\mathrm{b}}$} & Yes & 6773 & 53,892 & 98 & 1.10 & $0.89-1.35$ \\
\hline & No & 67,148 & 521,168 & 938 & 1.00 & - \\
\hline \multirow[t]{2}{*}{ Workers with higher socio economic status ${ }^{\mathrm{c}}$} & Yes & 6118 & 45,071 & 107 & 1.13 & $0.92-1.39$ \\
\hline & No & 41,182 & 289,793 & 659 & 1.00 & - \\
\hline \multirow[t]{2}{*}{ Workers with middle socio economic status ${ }^{\mathrm{c}}$} & Yes & 1599 & 13,099 & 45 & 1.55 & $1.14-2.11$ \\
\hline & No & 28,048 & 223,810 & 468 & 1.00 & - \\
\hline \multirow[t]{2}{*}{ Workers with lower socio economic status ${ }^{c}$} & Yes & 9600 & 80,388 & 253 & 1.00 & $0.88-1.15$ \\
\hline & No & 56,226 & 457,749 & 1473 & 1.00 & - \\
\hline \multirow[t]{2}{*}{ Workers with unknown socio economic status ${ }^{\mathrm{c}}$} & Yes & 2760 & 21,025 & 59 & 1.03 & $0.78-1.37$ \\
\hline & No & 14,400 & 107,064 & 310 & 1.00 & - \\
\hline
\end{tabular}

CI confidence interval

${ }^{\text {a }}$ The analysis was adjusted for calendar time, time passed since start of follow-up, age, weekly working hours, sex and socio-economic status

${ }^{\mathrm{b}}$ The analysis was adjusted for calendar time, time passed since start of follow-up, age, weekly working hours, and socio-economic status

${ }^{\mathrm{c}}$ The analysis was adjusted for calendar time, time passed since start of follow-up, age, weekly working, and sex

Table 3 Rate ratio for mortality among employees in Denmark 2000-2014 (protocol-based sensitivity analyses)

\begin{tabular}{llrrrrr}
\hline Population & Night-time work & Persons & Person years & Cases & Rate ratio & 95\% CI \\
\hline All workers & Regular & 9798 & 84,669 & 257 & 1.11 & $0.97-1.26$ \\
& Occasional & 10,279 & 74,914 & 207 & 1.04 & $0.90-1.20$ \\
& None & 139,856 & $1,078,416$ & 2910 & 1.00 & - \\
Workers interviewed more than once, & Yes & 8078 & 55,274 & 166 & 1.14 & $0.97-1.34$ \\
with stable night-work status & No & 96,708 & 627,228 & 1611 & 1.00 & - \\
Workers interviewed in 1999-2000 & Yes & 3700 & 51,754 & 183 & 1.06 & $0.91-1.25$ \\
& No & 22,416 & 316,067 & 1063 & 1.00 & - \\
Workers interviewed in 2001-2006 & Yes & 6327 & 65,024 & 196 & 1.17 & $1.00-1.36$ \\
& No & 40,671 & 419,397 & 1098 & 1.00 & - \\
Workers interviewed in 2007-2013 & Yes & 10,050 & 42,805 & 85 & 0.93 & $0.74-1.17$ \\
& No & 76,769 & 342,952 & 749 & 1.00 & - \\
\hline
\end{tabular}

The analyses were adjusted for calendar time, time passed since the start of follow-up, age, weekly working hours, sex and socio-economic status CI confidence interval 
In the first sensitivity analysis restricted to employees with a stable night work status, the rate ratio was estimated at 1.14 (95\% CI 0.97-1.34), which should be compared to the rate ratio of 1.07 that was obtained in the primary analysis (Table 3 ).

Although not statistically significant, the second sensitivity analysis found regular night-time work associated with higher mortality rates (RR $1.11,95 \%$ CI $0.97-1.26)$ than occasional night-time work (RR 1.04, 95\% CI 0.90-1.20) compared to no night-time work.

The third sensitivity analysis, which stratified rate ratios by calendar period, did not suggest any relation between the survey questions and the obtained rate ratios. The differences between the rate ratios from the three time periods were not greater than what is likely to occur from random variation and the rate ratios which differed the most, were obtained from the questions which differed the least (Table 3 ).

To test if the results were robust, we included three post-hoc analyses. First, we tested if the main results were affected by the presence of nurses, even though the analyses were adjusted for SES, by excluding all health personnel. The rate ratios (data not shown) were slightly higher but still close to the ones obtained in the primary analysis. In post-hoc analysis 2-3, the rate ratios among night workers in health care institutions, etc. were estimated at 0.93 (0.71-1.22) and 0.99 (0.79-1.25) with a full-time inclusion criterion at 32-100 and 28-100 h a week, respectively. The corresponding rate ratios among night workers in other sectors were estimated at $1.10(0.98-1.22)$ and $1.09(0.98-1.21)$.

Further data about the above mentioned sensitivity analyses are given in Tables 3 and 4.

\section{Discussion}

In the present study, we did not find any statistically significant relation between night-time work and all-cause mortality, neither as a general effect nor as an interaction effect with sex or socioeconomic status.
In the study, we were not able to include all relevant covariates in regard to mortality. We, therefore, included SES as a crude proxy for lifestyle factors, as lower socioeconomic position is associated with, e.g. smoking, alcohol intake, higher BMI, etc. (Marmot and Wilkinson 2005). Smoking and BMI are major risk factors for mortality (Aune et al. 2016; McEvoy et al. 2012) and it has previously been shown that the prevalence of smokers, as well as the prevalence of high BMI were higher among employees with than without night-time work in a random sample of our target population (Hannerz et al. 2016). The night workers were, in other words, expected to have higher mortality rates than the non-night workers, and so they did, although not statistically significant. It is, therefore, unlikely that the null-finding of the present study was due to a failure to control for lifestyle factors.

In our third sensitivity analysis, we stratified the results by interview period. The employees who participated in the surveys of 1999 or 2000 had the longest duration of follow-up (on average 14 years). The rate ratio for allcause mortality among the night-workers in this strata was estimated at 1.06 , which is very close to that obtained on the total sample $(\mathrm{RR}=1.07)$ where the average followup was 7.7 years. We, therefore, do not believe that the failure to find an effect was due to a too short duration of follow-up.

The main advantages of the present study are that the sample was large enough to afford an acceptable statistical power, that the participants were randomly selected from the target population and that the deaths and migrations were ascertained through national registers which cover all inhabitants of Denmark. Another advantage is that we are dealing with the outcome "death regardless of cause", which for all practical purposes is free from diagnostic errors.

The main drawback of the study is its lack of data on the history and duration of night-time work. Some of the participants who did not work at night during the 4 weeks preceding the interview might have been heavily exposed to night-time work both before and after the concerned 4-week
Table 4 Rate ratio for mortality among employees who worked between 28 and $100 \mathrm{~h}$ per week

\begin{tabular}{llrrrrl}
\hline Population & $\begin{array}{l}\text { Night- } \\
\text { time work }\end{array}$ & Persons & Person years & Cases & Rate ratio & 95\% CI \\
\hline All workers & Yes & 21,706 & 172,880 & 505 & 1.08 & $0.98-1.18$ \\
& No & 152,148 & $1,172,605$ & 3167 & 1.00 & - \\
Workers in health care, nursing & Yes & 5242 & 41,856 & 99 & 0.99 & $0.79-1.25$ \\
homes and similar institutions & No & 16,123 & 119,862 & 301 & 1.00 & - \\
Workers in other sectors & Yes & 16,464 & 131,024 & 406 & 1.09 & $0.98-1.21$ \\
& No & 136,025 & $1,052,743$ & 2866 & 1.00 & - \\
\hline
\end{tabular}

The analyses were adjusted for calendar time, time passed since the start of follow-up, age, weekly working hours, sex and socio-economic status

CI confidence interval 
period, which would bias the estimated effects of night-time work toward unity. To investigate this, a sensitivity analysis on stable night-time work was conducted. The rate ratio of the sensitivity test (RR 1.14, 95\% CI: 0.97-1.34) supports the suspicion of a bias towards null in the primary analysis, where the rate ratio was estimated at 1.07 (95\% CI 0.97-1.19).

Another drawback is the potential selection bias from non-response in the interviews. The low response rates are problematic; they decreased from $70 \%$ in 2002 to $53 \%$ in 2013. It has been shown that response rates to public health questionnaires in Denmark tend to be lower among unmarried people, young men, people with a low educational level and people with an ethnic background other than Danish (Christensen et al. 2014; Marmot and Wilcinson 2005). We cannot rule out the possibility that the response rates differ between the night workers and daytime workers. Neither can we rule out the possibility that the reasons for non-response differs between these groups. We know that the probability of non-response depends on age, sex and SES (Christensen et al. 2012, 2014). We also know that non-response tends to have a greater effect on prevalence estimates than it has on estimated associations (Cheung et al. 2017). We, therefore, believe that any such bias would have been mitigated by our decision to focus on relative rates rather than absolute rates and to control for age, sex, calendar period and SES.

The primary analyses used a dichotomised nighttime work variable (yes/no). As this could cover up a dose-response relationship, a sensitivity test was conducted including the three different response categories: regularly/ occasionally/no. Although statistically insignificant the analysis did suggest a dose-response relationship (regular night-time work: (RR 1.11, 95\% CI 0.97-1.26), occasional night-time work (RR 1.04, 95\% CI 0.90-1.20), no night-time work (the reference group)).

We found ten papers on cohort studies with estimated rate ratios for all-cause mortality among shift or night vs. daytime workers, one from Japan (Fujino et al. 2006), one from Great Britain (Taylor and Pocock 1972), two from USA (Gu et al. 2015; Kawachi et al. 1995), two from Denmark (Boggild et al. 1999; Jorgensen et al. 2017), two from Sweden (Akerstedt et al. 2004; Karlsson et al. 2005) and two from Germany (Oberlinner et al. 2009; Yong et al. 2014). In six of the papers (Boggild et al. 1999; Fujino et al. 2006; Karlsson et al. 2005; Oberlinner et al. 2009; Taylor and Pocock 1972; Yong et al. 2014), all of the reported estimates were close to unity and none was statistically significant. The null-finding of the present study is in line with these studies.

One of the 4 papers which reported rate ratios that were significantly different from unity dealt with a cohort of nurses from Denmark (Jorgensen et al. 2017), two dealt with a cohort of nurses from USA (Gu et al. 2015; Kawachi et al. 1995) and one dealt with a cohort that was randomly sampled from the general population of Sweden (Akerstedt et al. 2004).

The Swedish cohort study (Akerstedt et al. 2004) was based on a random sample of the Swedish population combined with the Swedish Cause-of-Death register. A total of 864 deaths were observed, during an average follow-up period of 11.8 years. The analyses were stratified by sex and socioeconomic status, and the results were adjusted for age, physically strenuous work, long-term disease, hectic work and smoking. The all-cause mortality rate ratio for selfreported "three-shift work, night work, evening work, roster work, and other forms" vs. "daytime work" was estimated at 1.04 (95\% CI 0.82-1.33) among male blue- and lowerlevel white-collar workers, 0.79 (95\% CI 0.50-1.26) among female blue- and lower-level white-collar workers, 1.23 (95\% CI 0.75-2.03) among male intermediate and higherlevel white-collar workers, and 2.61 (95\% CI 1.26-5.41) among female intermediate and higher-level white-collar workers. We note that the results of present study, where risk estimates were highest among those from the middle SES, do not agree with results of the Swedish cohort study (Akerstedt et al. 2004).

The US nurse cohort comprised 79,109 female nurses, who were 42-67 years old and free from diagnosed coronary heart disease and stroke at baseline in 1988. The cohort was examined for all-cause mortality by Kawachi et al. (1995), who observed 738 deaths during a follow-up period of 4 years. Compared with those who had never done shift work, the age-adjusted rate ratios for all-cause mortality were 0.91 (95\% CI 0.79-1.05) among those reporting $1-5$ years and 1.29 (95\% CI 1.10-1.52) among those reporting 6 or more years of rotating night shifts. The study by Kawachi et al. (1995) was repeated with an extended follow-up period (22 years) by Gu et al. (2015), who observed 14,181 deaths and age-adjusted rate ratios for all-cause mortality at 0.97 (95\% CI 0.94-1.01) among those reporting $1-5$ years, 1.19 (1.13-1.25) among those reporting 6-14 years and 1.24 (1.17-1.32) among those reporting 15 or more years of rotating night shifts. In the current study, we were not able to include years of exposure to night-time work, which is why we cannot confirm the findings from the above studies that indicated that more than five years of night-time work increases mortality rates.

The Danish nurse cohort comprised 18,015 female nurses in Denmark, who were gainfully employed and more than 44 years old at entry into the cohort (some entered in 1993 while others entered in 1999). The members of the cohort were followed for mortality from the time of their baseline interview (1993 or 1999) until 31 December 2012 by Jørgensen et al. (2017), who reported age-standardised rate ratios for all-cause mortality at 1.53 (95\% CI 1.33-1.77) for evening work, 1.77 (1.48-2.07) for night work and 0.98 (0.86-1.12) for rotating shift work, when compared with 
regular daytime work. The exposure categories were based on the question: "Do you normally work in: (a) day, (b) evening, (c) night, or (d) rotating shifts?".

It was the results obtained by Jorgensen et al. (2017) that prompted us to perform the present study; we wanted to know if the found association between night-time work and all-cause mortality among female nurses in Denmark could be reproduced among employees in the general working population of Denmark. Contrary to our expectations, the estimated rate ratios for night-time work in our post-hoc sensitivity analyses were lower among employees in the health care sector than they were among other employees. We note, however, that the apparent lack of agreement between the results of our post-hoc analyses and the results obtained by Jorgensen et al. may have been due to chance. We also note that the present study did not differentiate between rotating and fixed night shifts. Moreover, as the estimates from the current analysis are closest to the one of rotating shifts, there might be differences in how exposure is evaluated. Further, the increased awareness of health risks associated with night-time work may have changed night-time work habits and schedules, and the study by Jørgensen et al. concerned night-time work in 1993 or 1999 while the present study concerned night-time work in the time period 1999-2013.

The observed absence of an association with all-cause mortality does not preclude the possibility that night-time work may be associated with cause-specific mortality. Indeed Jorgensen et al. found an increased risk of mortality due to cardiovascular disease and diabetes, but not mortality due to cancer or psychiatric diseases (Jorgensen et al. 2017). Furthermore, night work has been associated increased risk of cardiovascular disease, breast and prostate cancer, diabetes, and gastrointestinal disorders (Hansen et al. 2016; Kivimaki et al. 2011; Knutsson and Boggild 2010; Torquati et al. 2018; Vyas et al. 2012) Thus, our findings do not imply that the current guidelines for organisation of night work (see e.g. Bonde et al. 2012). should be changed, and there are still good reasons to study how more specific shift characteristics are associated with health. Further studies of the topic could benefit of objective measurement of exposure, e.g. by payroll data as this may limit the risk of bias. Finally, other studies have found cause-specific mortality rates to be higher among night-time workers, which is why this could be included in future studies as well.

\section{Conclusion}

Our primary analyses did not find any statistically significant association between night-time work and all-cause mortality among employees in the general workforce of Denmark. It should, however, be noted that the absence of an association with all-cause mortality does not preclude the possibility that night-time work may be associated with cause-specific mortality.

Acknowledgements The data of the project were provided by Statistics Denmark. Marie Louise Kirkegaard, Sannie Vester Thorsen, Mads Nordentoft and Elizabeth Bengtsen at the National Research Centre for the Working Environment are thanked for guidance and valuable discussions.

Author contributions ADL initiated the study. HH and HSJ designed the study and wrote the study protocol. HH performed the statistical analyses. HSJ, HH and AHG prepared the first draft of the manuscript. All authors participated in a critical revision of the manuscript.

\section{Compliance with ethical standards}

Ethical approval The study complies with The Act on Processing of Personal Data, Denmark (Act No. 429 of May 31, 2000), which implements the European Union (EU) Directive 95/46/EC on the protection of individuals. Participation in the Labour Force Survey is voluntary. The data usage is approved by the Danish Data Protection Agency, file number 2001-54-0180. The ethical aspect of the project was examined and approved by Statistics Denmark, account number 704291. The data belong to Statistics Denmark and may only be used with their permission.

Informed consent By law, no informed consent is needed when using survey data.

Conflict of interest The authors declare that they have no conflict of interest.

Open Access This article is distributed under the terms of the Creative Commons Attribution 4.0 International License (http://creativeco mmons.org/licenses/by/4.0/), which permits unrestricted use, distribution, and reproduction in any medium, provided you give appropriate credit to the original author(s) and the source, provide a link to the Creative Commons license, and indicate if changes were made.

\section{References}

Akerstedt T, Kecklund G, Johansson SE (2004) Shift work and mortality. Chronobiol Int 21(6): 1055-1061

Aune D et al (2016) BMI and all cause mortality: systematic review and non-linear dose-response meta-analysis of 230 cohort studies with 3.74 million deaths among 30.3 million participants. BMJ 353:i2156. https://doi.org/10.1136/bmj.i2156

Boggild H, Suadicani P, Hein HO, Gyntelberg F (1999) Shift work, social class, and ischaemic heart disease in middle aged and elderly men; a 22 year follow up in the Copenhagen Male Study. Occup Environ Med 56(9):640-645

Bonde JP et al (2012) Work at night and breast cancer-report on evidence-based options for preventive actions. Scand J Work Environ Health 38(4):380-390. https://doi.org/10.5271/sjweh.3282

Cheung KL, Ten Klooster PM, Smit C, de Vries H, Pieterse ME (2017) The impact of non-response bias due to sampling in public health studies: a comparison of voluntary versus mandatory recruitment in a Dutch national survey on adolescent health. BMC Public Health 17(1):276. https://doi.org/10.1186/s12889-017-4189-8

Christensen AI et al (2012) The Danish National Health Survey 2010. Study design and respondent characteristics. Scand J Public 
Health 40(4):391-397. https://doi.org/10.1177/140349481245141 2

Christensen AI, Ekholm O, Glumer C, Juel K (2014) Effect of survey mode on response patterns: comparison of face-to-face and self-administered modes in health surveys. Eur J Public Health 24(2):327-332. https://doi.org/10.1093/eurpub/ckt067

Fujino Y et al (2006) A prospective cohort study of shift work and risk of ischemic heart disease in Japanese male workers. Am J Epidemiol 164(2):128-135

Gu F et al (2015) Total and cause-specific mortality of U.S. nurses working rotating night shifts. Am J Prev Med 48(3):241-252

Hannerz H, Soll-Johanning H (2017) General Mortality in relation to the EU Working Time Directive: a Danish study protocol. https:// figshare.com/articles/General_mortality_in_relation_to_the_EU_ Working_Time_Directive_a_Danish_study_protocol/5297062/1

Hannerz H, Larsen AD, Garde AH (2016) Working time arrangements as potential risk factors for Ischemic Heart Disease among workers in Denmark: a study protocol. JMIR Res Protoc 5(2):e130

Hansen AB, Stayner L, Hansen J, Andersen ZJ (2016) Night shift work and incidence of diabetes in the Danish Nurse Cohort. Occup Environ Med 73(4):262-268

Hedegaard NB, C (2014) Skab sunde arbejdstider. En vejledning om arbejdstid og arbejdsmiljø. FOA, Copenhagen

Itani O, Kaneita Y (2016) The association between shift work and health: a review. Sleep Biol Rhythms 14(3):231-239

Jorgensen JT, Karlsen S, Stayner L, Andersen J, Andersen ZJ (2017) Shift work and overall and cause-specific mortality in the Danish nurse cohort. Scand J Work Environ Health 43(2):117-126

Karlsson B, Alfredsson L, Knutsson A, Andersson E, Toren K (2005) Total mortality and cause-specific mortality of Swedish shift- and dayworkers in the pulp and paper industry in 1952-2001. Scand J Work Environ Health 31(1):30-35

Kawachi I et al (1995) Prospective study of shift work and risk of coronary heart disease in women. Circulation 92(11):3178-3182

Kecklund G, Axelsson J (2016) Health consequences of shift work and insufficient sleep. BMJ 355:i5210. https://doi.org/10.1136/ bmj.i5210.:i5210

Kivimaki M, Batty GD, Hublin C (2011) Shift work as a risk factor for future type 2 diabetes: evidence, mechanisms, implications, and future research directions. PLoS Med 8(12):e1001138. https://doi. org/10.1371/journal.pmed.1001138

Knutsson A, Boggild H (2010) Gastrointestinal disorders among shift workers. Scand J Work Environ Health 36(2):85-95

Lin X, Chen W, Wei F, Ying M, Wei W, Xie X (2015) Night-shift work increases morbidity of breast cancer and all-cause mortality: a meta-analysis of 16 prospective cohort studies. Sleep Med 16(11):1381-1387. https://doi.org/10.1016/j.sleep.2015.02.543

Marmot M, Wilcinson K (2005) Social determinants of health, 2nd edn. Oxford University Press, Oxford
Marmot M, Wilkinson R (2005) Social determinants of health, 2nd edn. Oxford University Press, Oxford

McEvoy JW et al (2012) Mortality rates in smokers and nonsmokers in the presence or absence of coronary artery calcification. JACC Cardiovasc Imaging 5(10):1037-1045

Oberlinner C et al (2009) Medical program for shift workers-impacts on chronic disease and mortality outcomes. Scand J Work Environ Health 35(4):309-318

Pedersen CB (2011) The Danish civil registration system. Scand J Public Health 39(7 Suppl):22-25

Petersson F, Baadsgaard M, Thygesen LC (2011) Danish registers on personal labour market affiliation. Scand J Public Health 39(7 Suppl):95-98

Smet M (1993) The Council of the European Union. Council Directive 93/104/EC of 23 November 1993 concerning certainaspects of the organization of working time. https://eur-lex.europa.eu/LexUr iServ/LexUriServ.do?uri=CELEX:31993L0104:EN:HTML

Statistics Denmark (1996) Dansk Branchekode 1993, 2.udgave. https ://www.dst.dk/Site/Dst/Udgivelser/GetPubFile .aspx?id=4829\&sid=dansk-branchekode-1993-hele-bogen

Statistics Denmark (2002) Dansk branchekode 2003-DB03. https ://www.dst.dk/Site/Dst/Udgivelser/GetPubFile .aspx $? \mathrm{id}=5132 \&$ sid $=$ hele 1

Statistics Denmark (2007) Dansk branchekode 2007-DB07. http:// www.dst.dk/Site/Dst/Udgivelser/GetPubFile.aspx?id=11119 \&sid=helepubl

Statistics Denmark (2015) Arbejdskraftundersøgelsen. https://www. dst.dk/da/Statistik/dokumentation/statistikdokumentation/arbej dskraftundersoegelsen

Taylor PJ, Pocock SJ (1972) Mortality of shift and day workers 195668. Br J Ind Med 29(2):201-207

Torquati L, Mielke GI, Brown WJ, Kolbe-Alexander T (2018) Shift work and the risk of cardiovascular disease. A systematic review and meta-analysis including dose-response relationship. Scand J Work Environ Health 44(3):229-238. https://doi.org/10.5271/ sjweh. 3700

Vyas MV et al (2012) Shift work and vascular events: systematic review and meta-analysis. BMJ 345:e4800. https://doi.org/10.1136/bmj. e4800.:e4800

Yong M, Nasterlack M, Messerer P, Oberlinner C, Lang S (2014) A retrospective cohort study of shift work and risk of cancer-specific mortality in German male chemical workers. Int Arch Occup Environ Health 87(2):175-183

Publisher's Note Springer Nature remains neutral with regard to jurisdictional claims in published maps and institutional affiliations. 